\title{
Evolution and Nucleosynthesis in Extremely Metal-Poor, Asymptotic Giant Branch Stars
}

\author{
Nobuyuki Iwamoto ${ }^{\mathrm{A}}$

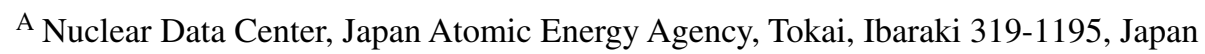 \\ Received 2008 November 15, accepted 2009 April 1
}

\begin{abstract}
We evolve extremely metal-poor $([\mathrm{Fe} / \mathrm{H}] \simeq-3)$, thermally pulsing Asymptotic Giant Branch (AGB) models with the mass range of $1-8 \mathrm{M}_{\odot}$. The chemical yields ejected from the models are obtained by considering mass loss. We find that the 1- and 2-M $\mathrm{M}_{\odot}$ AGB models are not affected by hot bottom burning (HBB). Nevertheless, they produce large amount of ${ }^{7} \mathrm{Li}$ in an $\mathrm{H}$-flash event. The occurrence of this event is associated with the ingestion of protons from the overlying H-rich envelope into the He convective shell driven by thermal pulse. The resulting ${ }^{7} \mathrm{Li}$ abundances in the ejecta are higher than the primordial one predicted in Big-Bang nucleosynthesis. The efficient production of ${ }^{7} \mathrm{Li}$ by the operation of $\mathrm{HBB}$ is also confirmed in the models of $4-8 \mathrm{M}_{\odot}$. If these AGB stars have a low-mass companion, it is probable that mass loss from the primary AGB star brings the materials enriched in ${ }^{7} \mathrm{Li}$ into the secondary star. This makes the surface composition of the secondary Li-rich. The formation of Li-rich stars, however, is strongly dependent on the mass loss history and binary separation. The nucleosynthesis for the other light nuclei is also calculated up to the end of the AGB phase. We find that the abundance patterns of the metal-poor stars CS 29528-041 and CS 29497-030 are well reproduced by yields from our AGB models.
\end{abstract}

Keywords: stars: AGB and post-AGB — nuclear reactions, nucleosynthesis, abundances - stars: evolution - stars: mass loss

\section{Introduction}

After the end of core He-burning phase, low and intermediate-mass (e.g. $\sim 0.8-10 \mathrm{M}_{\odot}$ ) stars enter into the thermally pulsing asymptotic giant branch (TP-AGB) phase (e.g. Herwig 2005). In this phase the structure of AGB stars has electron-degenerate carbon-oxygen (or oxygen-neon) core, the surrounding thin He-rich layer and the outer H-rich envelope. The He shell burning takes place at the bottom of the He layer. This burning shell is thermally unstable and recurrently induces thermal runaway, which is called thermal pulse (or He shell flash). The He shell convection develops in He layer during thermal pulse phase. The extension of the convective shell is terminated by the existence of a large entropy difference at the boundary between H-rich envelope and He layer. The convective mixing distributes newly synthesized nuclei over the He layer. After the thermal pulse ends, the envelope convection penetrates into the He buffer layer where the He convective shell develops and brings the produced nuclei (e.g. ${ }^{12} \mathrm{C}$ and $s$-process elements) into the surface (i.e. the third dredge-up). This leads to the enhancement of ${ }^{12} \mathrm{C}$ and $s$-process elements in the surface of AGB stars. On the other hand, another important nucleosynthetic process occurs in luminous intermediate-mass AGB stars (e.g. Wood, Bessell \& Fox 1983; Plez, Smith \& Lambert 1993). The base of the convective envelope reaches the H-burning shell. The H-burning directly processes the nuclei in the convective envelope during interpulse phases (hot bottom burning, $\mathrm{HBB}$ ). The dredged-up ${ }^{12} \mathrm{C}$ and ${ }^{16} \mathrm{O}$ are converted into ${ }^{14} \mathrm{~N}$ via the $\mathrm{CNO}$ cycle. This results in making the surface N-rich. As the result, a variety of chemical elements with large enhancements has been observed in the surface of AGB stars (Lambert et al. 1995).

Fujimoto, Ikeda \& Iben (2000) categorized the initial mass-metallicity range by considering the evolutionary characteristics. In Case $\mathrm{II}^{\prime}$ which corresponds to $[\mathrm{Fe} / \mathrm{H}] \lesssim-2.5$ and $1 \lesssim M / M_{\odot} \lesssim 3$ the evolution of AGB stars undergoes an H-ingestion event (HIE, Fujimoto et al. 2000; Iwamoto et al. 2004; Suda et al. 2004; Straniero et al. 2004; Campbell \& Lattanzio 2008). Since in the Case-II' evolution the entropy barrier between the $\mathrm{H}$-rich envelope and He-rich layer is low, the He convective shell developing during thermal pulse is able to penetrate into the overlying H-rich envelope. The convective mixing carries protons into the hotter He-burning region. The ingested protons are captured by ${ }^{12} \mathrm{C}$ (produced by the triple $\alpha$ reaction) at the location where the mixing timescale is comparable to the timescale of proton capture on ${ }^{12} \mathrm{C}$. This reaction releases a huge amount of energy and leads to an H-flash. The He convective shell is split by the energy release into two convective shells: one is driven by the H-flash and another is driven by the He-flash. Soon after the H-flash ceases, the envelope convection penetrates into the region where the $\mathrm{H}$-flash driven convective shell is developed. The produced ${ }^{14} \mathrm{~N}$ and ${ }^{13} \mathrm{C}$ as well as ${ }^{12} \mathrm{C}$ are brought into the envelope. This HIE 
makes the surface of low-mass, low-metallicity AGB stars $\mathrm{CN}$-rich.

It has been considered that lithium is one of the few elements which are primordially produced by big-bang nucleosynthesis. The primordial abundance was analyzed from the power spectrum of the cosmic microwave background fluctuations taken by the WMAP satellite and was determined to be $A(\mathrm{Li})^{1}=2.64$ (Spergel et al. 2003, 2007). The value is slightly higher than the Spite plateau value of $A(\mathrm{Li})=2.1$ to 2.2 , which was observed in metalpoor, warm dwarfs (Spite \& Spite 1982). This discrepancy might be attributable to the decrease in Li abundance by, for example, atomic diffusion (e.g. Richard, Michaud \& Richer 2005), rotation-induced mixing (e.g. Talon \& Charbonnel 1998) and thermohaline mixing (e.g. Stancliffe et al. 2007; Stancliffe \& Glebbeek 2008) in the long-lived dwarfs, since ${ }^{7} \mathrm{Li}$ is a fragile nuclide and ${ }^{7} \mathrm{Li}(p, \alpha)$ reaction becomes active at relatively low temperature of $2.5 \times 10^{6} \mathrm{~K}$.

The HBB contributes to the production of ${ }^{7} \mathrm{Li}$ in the convective envelope of intermediate-mass AGB stars in which the base temperature is higher than $5 \times 10^{7} \mathrm{~K}$. The ${ }^{3} \mathrm{He}(\alpha, \gamma)$ reaction firstly creates ${ }^{7} \mathrm{Be}$ at the base of the convective envelope where the timescale of ${ }^{7} \mathrm{Li}(p, \alpha)$ reaction is very short. Fortunately, part of the produced ${ }^{7} \mathrm{Be}$ is convectively transported into the cool outer envelope where the ${ }^{7} \mathrm{Li}(p, \alpha)$ reaction is not active. The ${ }^{7} \mathrm{Be}$ decays to ${ }^{7} \mathrm{Li}$ via the ${ }^{7} \mathrm{Be}\left(\mathrm{e}^{-}, v_{e}\right)$ reaction. Although the ${ }^{7} \mathrm{Li}$ produced in the cool envelope is brought into the hotter H-burning shell where the ${ }^{7} \mathrm{Li}(p, \alpha)$ reaction works efficiently, ${ }^{7} \mathrm{Li}$ is progressively accumulated in the outer envelope. This process of $\mathrm{Li}$ production has been called beryllium transport mechanism (Cameron \& Fowler 1971) and its operation was firstly confirmed by Sackmann \& Boothroyd (1992) numerically. Their results explained the high Li abundances $(A(\mathrm{Li}) \sim 4)$ observed at the surface of luminous AGB stars in Large and Small Magellanic Clouds (Smith \& Lambert 1989, 1990).

The large depletion of surface ${ }^{7} \mathrm{Li}$ abundance in HE1327-2326, which is one of the hyper metal-poor (HMP) stars ever known, was recognized (Frebel et al. 2005, 2008). Tumlinson (2007) argued that this observational fact favors the mass-transfer scenario in low-mass binary, since $\mathrm{Li}$ is not contained in the accreting gases which is mixed with the convective envelope of the secondary star with the primordial Li abundance. Suda et al. (2004) discussed the binary scenario, in order to explain the origin of the surface abundance patterns of HE01075240 (Christlieb et al. 2002). Without taking into account the ${ }^{7} \mathrm{Li}$ production in AGB stars, they concluded that the abundance pattern of the light elements is attributed to the yields ejected during an AGB phase of the primary star with mass less than $2.5 \mathrm{M}_{\odot}$. The $\mathrm{Li}$ abundance in the materials which are accreted onto the dwarf from the massive companion are changed by dilution in the shallow surface

${ }^{1} A(\mathrm{Li})=\log \left(N_{\mathrm{Li}} / N_{\mathrm{H}}\right)+12$ and $N$ is the number abundance of the element. convective zone. In addition, the other extra mixing processes mentioned above carry the accreted material much deeper. This makes the surface $\mathrm{Li}$ abundance decreased. In the case of thermohaline mixing, Stancliffe et al. (2007) concluded that the accreted materials with high mean molecular weight should be mixed with about $90 \%$ of the secondary. If the extra mixing brings the accreted materials into the region where the temperature is higher than $2.5 \times 10^{6} \mathrm{~K}$, the ${ }^{7} \mathrm{Li}$ in the accreted materials is destroyed by the $(p, \alpha)$ reaction. These may lead to the sufficient depletion of $\mathrm{Li}$.

In this paper we follow the evolution of low and intermediate-mass, TP-AGB stars with mass loss, and calculate the ejected yields of ${ }^{7} \mathrm{Li}$ and the other light nuclei. The influences of binary mass transfer from the primary low-metallicity AGB star on the surface chemical composition of the secondary star now observed are investigated.

\section{Stellar Models}

We evolve stellar models with mass loss from zero-age main sequence (ZAMS) up to the end of AGB phase and calculate the nucleosynthesis through the evolution. The effects of stellar rotation and overshooting are not taken into account in these calculations. The grid of our models covers the mass range of $M_{\text {ZAMS }}=1$ to $8 \mathrm{M}_{\odot}$. The heavy-element abundance $Z$ is adopted to be $2 \times 10^{-5}$ which corresponds to the metallicity of $[\mathrm{Fe} / \mathrm{H}]^{2} \simeq-3$. For the initial composition we assume the scaled solar abundances (Anders \& Grevesse 1989) without $\alpha$-element enhancements. The initial abundance of ${ }^{7} \mathrm{Li}$ is set to be zero. This assumption may be justified for the following reason. Since the first and/or second dredge-up will effectively bring the Li-depleted materials into the envelope before reaching TP-AGB phase, this leads to the significant reduction of surface ${ }^{7} \mathrm{Li}$ abundance, for example, by two orders of magnitude at the beginning of AGB phase (Forestini \& Charbonnel 1997). Therefore, this assumption probably doesn't matter for the Li yields, which mainly comes from the mass loss during AGB phases. As the solar system abundance of $\mathrm{Li}$ we take $A(\mathrm{Li})_{\odot}=3.31$ (Anders \& Grevesse 1989), when comparing our results with the observations.

Mass loss is an important factor to consider the evolution of AGB stars and the compositions of the yields ejected from the stellar surface. However, the dependence of the mass loss rate on chemical compositions is poorly known for extremely metal-poor AGB stars. Therefore, we first apply the Reimers mass-loss rate to the evolution from the ZAMS and then change it to the rate formulated by Blöcker (1995) when the Blöcker rate becomes higher than the Reimers' one. The Blöcker rate is based on the numerical simulation of dust-driven mass loss from the atmosphere of a low-mass star with long-period pulsation

\footnotetext{
${ }^{2}[\mathrm{~A} / \mathrm{B}]=\log \left(N_{\mathrm{A}} / N_{\mathrm{B}}\right)-\log \left(N_{\mathrm{A}} / N_{\mathrm{B}}\right)_{\odot}$, where the subscript $\odot$ refers to the value as the solar abundance.
} 
and is described by the following form:

$$
\dot{M}_{\mathrm{B}}=\eta_{\mathrm{B}} 4.83 \times 10^{-9} M_{\mathrm{ZAMS}}^{-2.1} L^{2.7} \dot{M}_{\mathrm{R}},
$$

where $L$ is the surface luminosity in solar unit and $\dot{M}_{\mathrm{R}}$ is the Reimers mass-loss rate which is multiplied by a factor of $\eta_{\mathrm{R}}=0.5$. However, we assume $\eta_{\mathrm{R}}=0.3$ in the $1-\mathrm{M}_{\odot}$ model, in order not to lost the envelope mass significantly before the ignition of core He burning, at which the abundance of heavy elements in the envelope are still low even after the first dredge-up. The factor $\eta_{\mathrm{B}}$ is further introduced to take into account the effects of the low-metal content on the Blöcker rate. In this work we set $\eta_{\mathrm{B}}=0.1$ (Herwig 2004). This adopted $\eta_{\mathrm{B}}$ value might be large at the early phase of the AGB evolution in the low metallicity condition. The resulting rate is by a factor of $0.05(0.03$ for the $1-\mathrm{M}_{\odot}$ model) smaller than the original Blöcker one. We note that the transition from the Reimers to the Blöcker rate occurs during the early AGB phase where surface luminosity increases rapidly.

The internal change of chemical composition over all evolutionary phases is followed by a technique in which convective mixing and nuclear processing are solved simultaneously. The mixing length theory is used for the convective mixing and energy transport. The convective boundary is determined by the Schwarzschild criterion. The reaction network consists of 56 nuclear species ranging from neutron and proton to sulfur. Nuclear reaction rates are mainly taken from Angulo et al. (1999), Bao et al. (2000), Rauscher \& Thielemann (2000) and Caughlan \& Fowler (1988). We have used the OPAL opacity tables for temperatures higher than $10^{4} \mathrm{~K}$ (Iglesias \& Rogers 1996) and the opacity tables given by Alexander \& Ferguson (1994) for the lower temperatures, assuming fixed $Z$. Marigo (2002) and Cristallo et al. (2007) showed that the effects of variable CNO abundances on the lowtemperature molecular opacity have a significant influence on the evolution of AGB stars. The molecular opacity with variable $\mathrm{CNO}$ abundances is not taken into account in the present calculations. However, this has almost no or a small influence on the HIE because the HIE occurs at the early phase of AGB evolution. In the later evolution where CNO abundance in the envelope is high due to HIE, recurrent third dredge-up, or both, the enhanced molecular opacity will affect the total number of thermal pulses (i.e. mass-loss rate) and, thus, the yield from AGB stars.

The other details on the stellar-evolution code can be found in Iwamoto et al. (2004).

\section{Results}

Figure 1 shows the evolutionary tracks of 1-, 2-, 3-, 4-, 6- and $8-\mathrm{M}_{\odot}$ models from ZAMS up to the final phase of AGB evolution. We note, however, that the envelope mass $\left(0.6-\mathrm{M}_{\odot}\right)$ is still present in the $8-\mathrm{M}_{\odot}$ model when the calculation is terminated. The models with $M>2 \mathrm{M}_{\odot}$ ignites the core He burning before ascending the red giant branch (RGB). Therefore, until the early AGB phases, the surface chemical compositions in these models have no

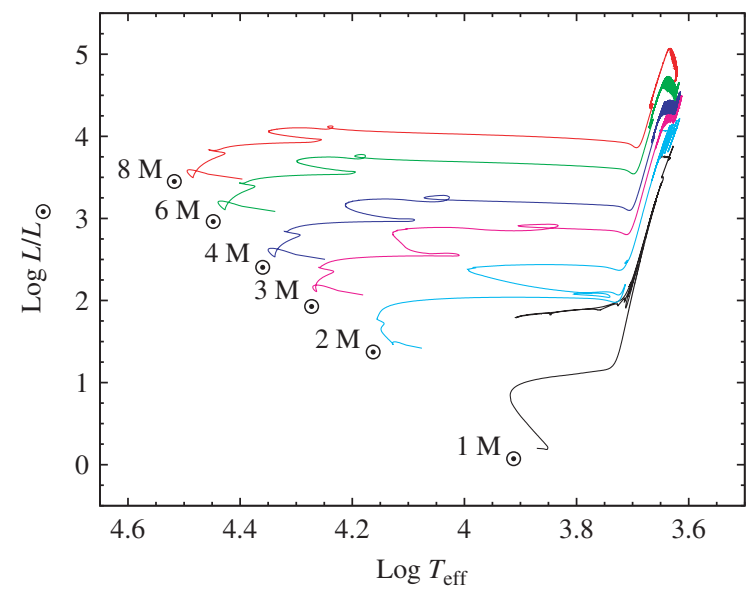

Figure 1 Evolutionary tracks of $1-8 \mathrm{M}_{\odot}$ models with $Z=2 \times$ $10^{-5}$ are shown from zero-age main sequence up to the end of AGB phase. The effective temperature-luminosity variations at the most luminous stage of each evolutionary track are caused by the occurrence of thermal pulses and mass loss.

influence from the deep interior where H-burning occurs. In contrast, the $1-\mathrm{M}_{\odot}$ model evolves to the RGB and experiences efficient mass loss with increase in luminosity in the RGB phase. At the beginning of the core He-burning phase this model undergoes an off-center He ignition (He core flash). After He in the convective core is depleted, it evolves to the TP-AGB phase under the current assumptions. We, however, notice that uncertain mass loss rate gives a significant impact on the later evolution. If the mass loss rate is higher than the present one, the evolution of AGB phase is bypassed and the resulting yield becomes very different. In the $8-\mathrm{M}_{\odot}$ model carbon-burning $(\mathrm{C}$ burning) begins off-center and forms an oxygen-neon core (for detailed evolution of intermediate-mass stars at the C-burning phase, see Ritossa, Garcia-Berro \& Iben 1996; Garcia-Berro, Ritossa \& Iben 1997; Iben, Ritossa \& Garcia-Berro 1997). After the central C-burning phase, this model also evolves toward the double-shell-burning AGB phase. The luminous AGB evolution is characterized by small effective temperature-luminosity variations of the tracks as seen in Figure 1. These are attributed to the occurrence of thermal pulses and mass loss.

The surface abundance variations of ${ }^{12} \mathrm{C},{ }^{14} \mathrm{~N},{ }^{16} \mathrm{O}$, ${ }^{22} \mathrm{Ne}$ and ${ }^{23} \mathrm{Na}$ in the calculated models are presented in Figure 2. The first and rapid increase of ${ }^{12} \mathrm{C},{ }^{14} \mathrm{~N}$ and ${ }^{16} \mathrm{O}$ abundances in the $1-$ and $2-\mathrm{M}_{\odot}$ models is induced by the HIE, which occurs only once because the high CNO abundances after the event form a large entropy barrier between the H-rich envelope and He-rich layer (Iwamoto et al. 2004). The enhancements of CNO nuclei lead to the drastic increase in surface luminosity and radius and, thus, in mass loss rate. The $1-\mathrm{M}_{\odot}$ model loses the envelope mass before the development of the next thermal pulse and leaves the AGB. In contrast, since the large envelope mass is left in the $2-\mathrm{M}_{\odot}$ model after the HIE, this model experiences thermal pulses and the following third dredge-ups 

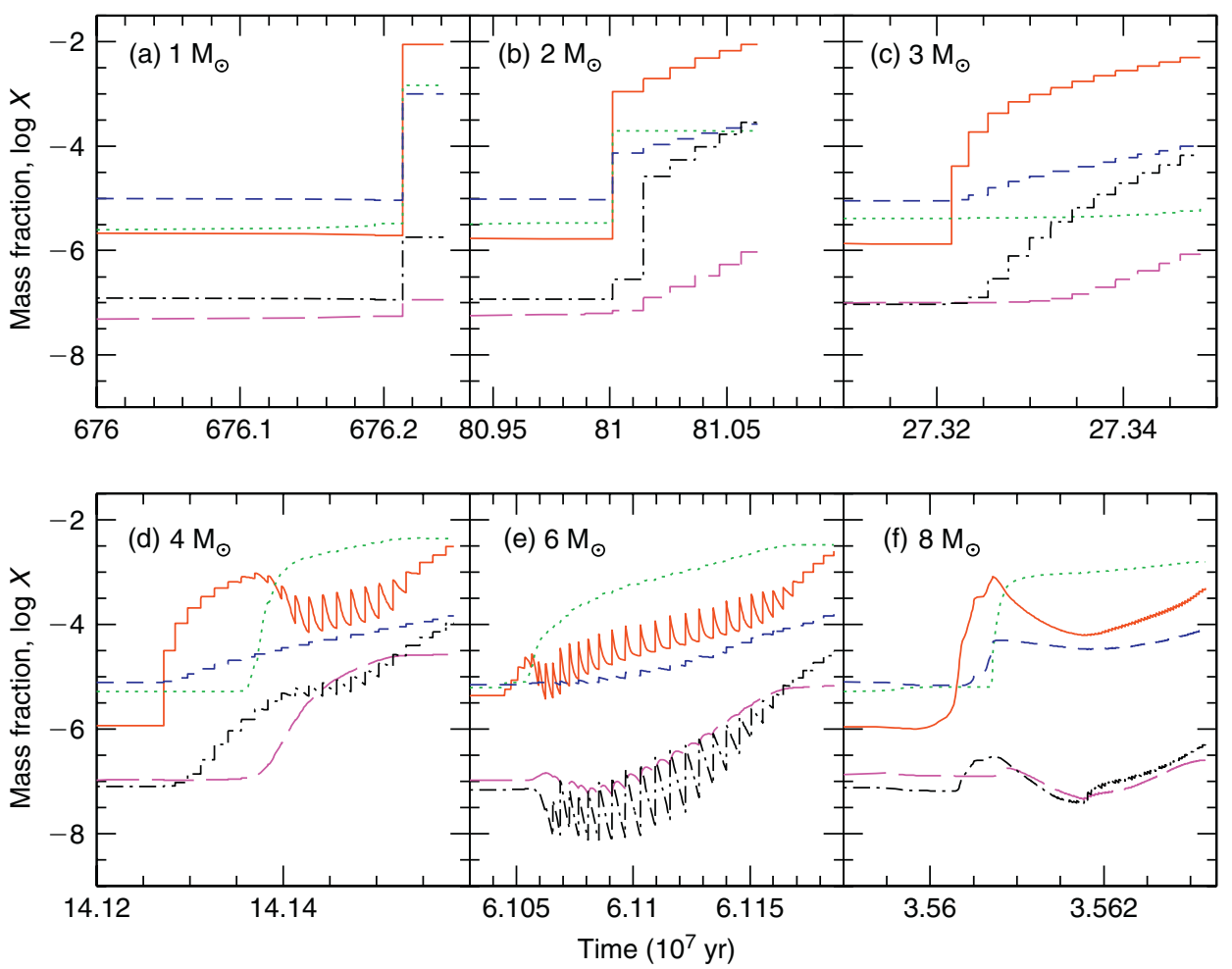

Figure 2 Surface variations of ${ }^{12} \mathrm{C}$ (solid lines), ${ }^{14} \mathrm{~N}$ (dotted lines), ${ }^{16} \mathrm{O}$ (dashed lines), ${ }^{22} \mathrm{Ne}$ (dash-dotted lines) and ${ }^{23} \mathrm{Na}$ (long-dashed lines) abundances (by mass) for models of $1-8 \mathrm{M}_{\odot}$, during TP-AGB phases. The occurrence of HIE is identified by the first increase of ${ }^{14} \mathrm{~N}$ as well as ${ }^{12} \mathrm{C}$ and ${ }^{16} \mathrm{O}$ in models of $1-$ and $2-\mathrm{M}_{\odot}$.

repeatedly. This leads to the additional enhancement of ${ }^{12} \mathrm{C}$ and ${ }^{16} \mathrm{O}$. Nevertheless, the ${ }^{14} \mathrm{~N}$ abundance remains unchanged.

The evolution of AGB models with $M \geq 3 \mathrm{M}_{\odot}$ does not experience the HIE. The third dredge-up progressively enriches the envelope with ${ }^{12} \mathrm{C}$ and ${ }^{16} \mathrm{O}$. In the models with $M>3 \mathrm{M}_{\odot}$ the $\mathrm{HBB}$ is gradually activated with the increase of the $\mathrm{H}$-exhausted core mass. The previously dredged-up ${ }^{12} \mathrm{C}$ is transmuted into ${ }^{14} \mathrm{~N}$ during interpulse phases as seen in Figure 2d-f. The conversion efficiency becomes low as the envelope masses are decreased by mass loss. As the result, the dredged-up ${ }^{12} \mathrm{C}$ survives the proton captures and, thus, the surface ${ }^{12} \mathrm{C}$ abundances increase again at the later phase of AGB evolution.

The large enhancement of ${ }^{22} \mathrm{Ne}$ is found in the models of $2-6 \mathrm{M}_{\odot}$. The ${ }^{22} \mathrm{Ne}$ is produced in the He convective shell by two $\alpha$ captures via the reactions ${ }^{14} \mathrm{~N}(\alpha, \gamma){ }^{18} \mathrm{~F}$ $\left(\beta^{+} \nu\right)^{18} \mathrm{O}(\alpha, \gamma)^{22} \mathrm{Ne}$. The ${ }^{14} \mathrm{~N}$ is the ash of $\mathrm{CNO}$ cycle left behind by the outward advancement of the H-burning shell. The third dredge-up brings the synthesized ${ }^{22} \mathrm{Ne}$ into the envelope. The ${ }^{22} \mathrm{Ne}$ in the envelope convection is converted into ${ }^{23} \mathrm{Na}$ via $\mathrm{NeNa}$ cycle of the H-burning by the operation of HBB (Mowlavi 1999). Therefore, the successive enrichment of ${ }^{23} \mathrm{Na}$ is obtained in the 4- and 6- $\mathrm{M}_{\odot}$ models. Figure 2 shows that the production efficiency of ${ }^{23} \mathrm{Na}$ reduces as the base temperature of convective envelope decreases. This fact is also found in the case of ${ }^{14} \mathrm{~N}$. In the lower mass models the ${ }^{22} \mathrm{Ne}(n, \gamma)$ reaction in the He convective shell is important to produce ${ }^{23} \mathrm{Na}$, which is dredged-up into the envelope together with ${ }^{22} \mathrm{Ne}$.
The surface variations of ${ }^{7} \mathrm{Li}$ abundances are shown in Figure 3. As explained above, the production of ${ }^{7} \mathrm{Li}$ occurs via the Be transport mechanism in the AGB models with $M \gtrsim 3 \mathrm{M}_{\odot}$. The models of $4-8 \mathrm{M}_{\odot}$ predict ${ }^{7} \mathrm{Li}$ abundances higher than the solar system abundance. The decrease in ${ }^{7} \mathrm{Li}$ abundances after the maximum value, is attributed to the consumption of ${ }^{3} \mathrm{He}$ contained in the convective envelope because ${ }^{3} \mathrm{He}$ is the primary source for the production of ${ }^{7} \mathrm{Li}$. The maximum ${ }^{7} \mathrm{Li}$ abundance in the $3-\mathrm{M}_{\odot}$ model is lower than that in the models of $4-8 \mathrm{M}_{\odot}$ by two orders of magnitude, since the bottom temperature of the convective envelope is much lower than that attained in the higher mass models. In contrast to the higher mass models, the ${ }^{7} \mathrm{Li}$ abundance in the $3-\mathrm{M}_{\odot}$ model decreases from the maximum value, though an adequate amount of ${ }^{3} \mathrm{He}$ is still present in the convective envelope. This is responsible for the bottom temperature of the envelope convection which is gradually decreases as the envelope mass is lost.

Since low mass (e.g. $M \lesssim 3 \mathrm{M}_{\odot}$ whose upper limit is changed by the metallicity and stellar input physics), AGB stars do not experience efficient HBB, they are hard to contribute to the production of ${ }^{7} \mathrm{Li}$ in $[\mathrm{Fe} / \mathrm{H}] \gtrsim-2.5$. $^{3}$

\footnotetext{
${ }^{3}$ The presence of Li-enriched, low-mass, metal-rich AGB stars has been known (Abia \& Isern 1997; Uttenthaler et al. 2007). However, the proposed explanation for the Li-richness is still a matter for debate (e.g. cool bottom processing, accretion of brown dwarfs and planets and mass transfer from a former intermediate-mass AGB companion). If the cool bottom processing is attributable to the enhancement of $\mathrm{Li}$, it is likely that some of low-mass AGB stars with appropriate conditions contribute to the ${ }^{7} \mathrm{Li}$ production.
} 

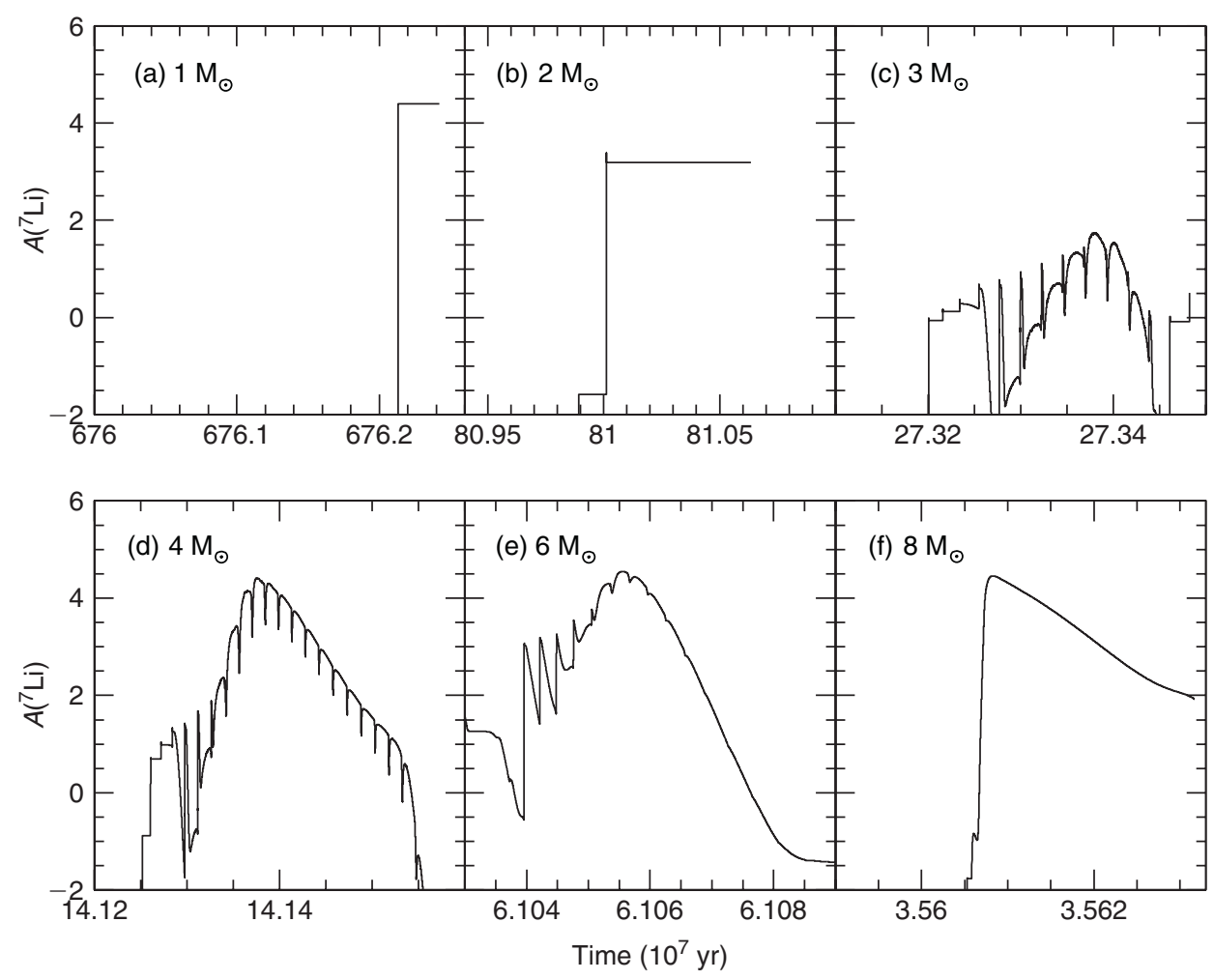

Figure 3 Surface variations of $\mathrm{Li}$ abundances in AGB stars of $1-8 \mathrm{M}_{\odot}$.

Table 1. Yields for light nuclides

\begin{tabular}{lccccc}
\hline$M_{\text {ZAMS }}$ & $1 \mathrm{M}_{\odot}$ & $2 \mathrm{M}_{\odot}$ & $3 \mathrm{M}_{\odot}$ & $4 \mathrm{M}_{\odot}$ & $6.84 \mathrm{M}_{\odot}$ \\
$M_{\alpha}{ }^{\mathrm{a}}$ & $0.53 \mathrm{M}_{\odot}$ & $0.66 \mathrm{M}_{\odot}$ & $0.81 \mathrm{M}_{\odot}$ & & $0.97 \mathrm{M}_{\odot}$ \\
\hline Nuclide & & & Yields $\left(\mathrm{M}_{\odot}\right)$ & & \\
\hline${ }^{7} \mathrm{Li}$ & $3.83 \times 10^{-08}$ & $9.76 \times 10^{-09}$ & $7.68 \times 10^{-11}$ & $2.72 \times 10^{-08}$ & $3.62 \times 10^{-09}$ \\
${ }^{12} \mathrm{C}$ & $3.06 \times 10^{-03}$ & $7.22 \times 10^{-03}$ & $6.18 \times 10^{-03}$ & $3.01 \times 10^{-03}$ & $1.86 \times 10^{-03}$ \\
${ }^{13} \mathrm{C}$ & $6.91 \times 10^{-04}$ & $3.28 \times 10^{-04}$ & $1.45 \times 10^{-06}$ & $2.98 \times 10^{-04}$ & $1.75 \times 10^{-04}$ \\
${ }^{14} \mathrm{~N}$ & $4.92 \times 10^{-04}$ & $2.46 \times 10^{-04}$ & $1.08 \times 10^{-05}$ & $9.67 \times 10^{-03}$ & $7.48 \times 10^{-03}$ \\
${ }^{16} \mathrm{O}$ & $3.49 \times 10^{-04}$ & $2.47 \times 10^{-04}$ & $1.31 \times 10^{-04}$ & $2.50 \times 10^{-04}$ & $2.11 \times 10^{-04}$ \\
${ }^{19} \mathrm{~F}$ & $3.57 \times 10^{-10}$ & $7.72 \times 10^{-07}$ & $1.73 \times 10^{-07}$ & $1.22 \times 10^{-07}$ & $2.49 \times 10^{-09}$ \\
${ }^{20} \mathrm{Ne}$ & $7.85 \times 10^{-07}$ & $3.29 \times 10^{-06}$ & $4.55 \times 10^{-06}$ & $8.26 \times 10^{-06}$ & $1.65 \times 10^{-05}$ \\
${ }^{22} \mathrm{Ne}$ & $6.51 \times 10^{-07}$ & $1.89 \times 10^{-04}$ & $6.23 \times 10^{-05}$ & $1.02 \times 10^{-04}$ & $2.18 \times 10^{-05}$ \\
${ }^{23} \mathrm{Na}$ & $4.44 \times 10^{-08}$ & $6.47 \times 10^{-07}$ & $8.92 \times 10^{-07}$ & $5.04 \times 10^{-05}$ & $9.60 \times 10^{-06}$ \\
\hline
\end{tabular}

${ }^{\mathrm{a}} \mathrm{H}$-exhausted core mass.

However, AGB stars in the Case-II and Case-II' regions classified by Fujimoto et al. (2000) can also create ${ }^{7} \mathrm{Li}$. The production is closely related with the occurrence of the HIE. At the onset of the thermal pulse involving the HIE, the extending He convective shell reaches the bottom of the H-rich envelope. The convection brings ${ }^{3} \mathrm{He}$ together with protons into the convective shell. As the result, in an $\mathrm{H}$-flash-driven convective shell formed just after the proton ingestion, ${ }^{7} \mathrm{Be}$ is efficiently created by the ${ }^{3} \mathrm{He}(\alpha, \gamma)$ reaction and is accumulated in the shell. Soon after the H-flash terminates, the outer-envelope convection penetrates into the former $\mathrm{H}$-flash layer and dredges up ${ }^{7} \mathrm{Be}$ from the layer. The ${ }^{7} \mathrm{Li}$ produced by the electron capture on ${ }^{7} \mathrm{Be}$ is finally distributed over the envelope.
After this event the bottom temperature of the convective envelope is too low to activate the ${ }^{7} \operatorname{Li}(p, \alpha)$ reaction. The surface ${ }^{7} \mathrm{Li}$ abundances of the 1 - and $2-\mathrm{M}_{\odot}$ models are not affected in the later AGB evolution during interpulse phases as seen in Figures $3 \mathrm{a}$ and $3 \mathrm{~b}$. It is found that the ejected amounts of ${ }^{7} \mathrm{Li}$ are large enough to influence the surface Li abundance of the binary companion even in the low mass models (Table 1). We note that there exists the mass range of $2-3 \mathrm{M}_{\odot}$ in which the extremely metalpoor stars may undergo production of ${ }^{7} \mathrm{Li}$ via $\mathrm{HIE}$ and destruction via HBB (Iwamoto et al. 2004).

Figure 4 shows ejected amounts of ${ }^{12} \mathrm{C},{ }^{14} \mathrm{~N},{ }^{16} \mathrm{O},{ }^{19} \mathrm{~F}$, ${ }^{22} \mathrm{Ne}$ and ${ }^{23} \mathrm{Na}$ in the calculated models. For comparison, the yield in the $8-\mathrm{M}_{\odot}$ model is calculated by assuming that 


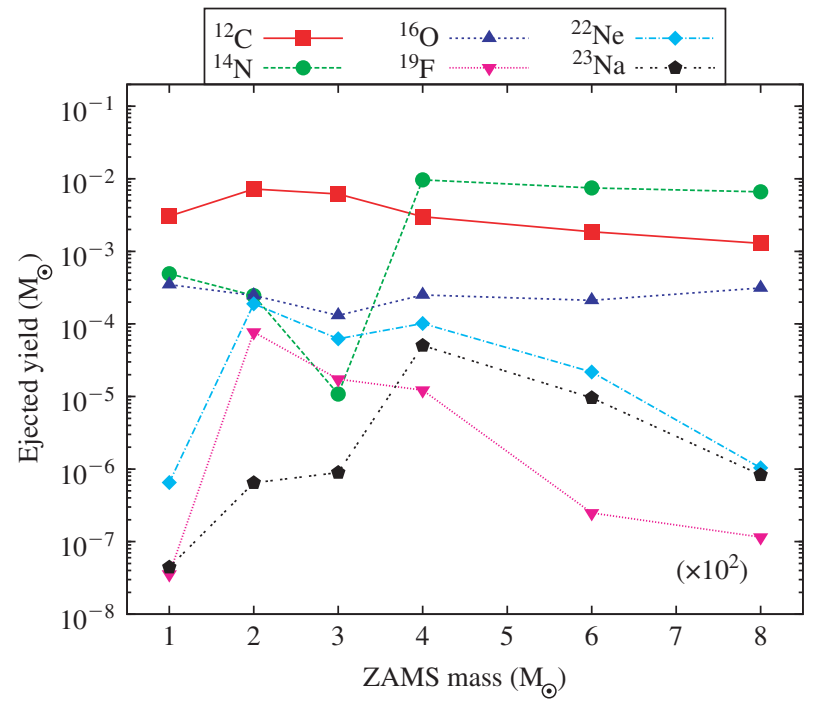

Figure 4 Ejected yields of ${ }^{12} \mathrm{C},{ }^{14} \mathrm{~N},{ }^{16} \mathrm{O},{ }^{19} \mathrm{~F},{ }^{22} \mathrm{Ne}$ and ${ }^{23} \mathrm{Na}$ in the AGB models. The amount of ${ }^{19} \mathrm{~F}$ is multiplied by 100 .

the remaining envelope mass is lost instantly as simple approximation. This may have an influence on the final yields of especially ${ }^{12} \mathrm{C}$. The ejected yields (in units of $\mathrm{M}_{\odot}$ ) for $\mathrm{CNO}$ and NeNa isotopes and ${ }^{7} \mathrm{Li}$, the ZAMS mass ( $\left.M_{\text {ZAMS }}\right)$, and H-exhausted core mass $\left(M_{\alpha}\right)$ at the end of AGB phase are presented in Table 1 . The yields of ${ }^{12} \mathrm{C}$ have a weak dependence on the stellar mass and exceed $10^{-3} \mathrm{M}_{\odot}$ in all models. In the models with $1-2 \mathrm{M}_{\odot}$, the ${ }^{14} \mathrm{~N}$ yields are large in comparison with the $3-\mathrm{M}_{\odot}$ model without HIE, but do not exceed those of ${ }^{12} \mathrm{C}$. The ejected ${ }^{14} \mathrm{~N}$ amounts in the models with $M \geq 4 \mathrm{M}_{\odot}$ are larger than those of ${ }^{12} \mathrm{C}$ due to the efficient production by the HBB. The ${ }^{16} \mathrm{O}$ yields are not sensitive to the initial mass and are the order of $10^{-4} \mathrm{M}_{\odot}$. The ejected masses of ${ }^{22} \mathrm{Ne}$ have a correlation with those of ${ }^{23} \mathrm{Na}$ in the $M \geq 4 \mathrm{M}_{\odot}$ models as shown in Figure $2 \mathrm{~d}-\mathrm{f}$. The 2- and $3-\mathrm{M}_{\odot}$ models also eject large amounts of ${ }^{22} \mathrm{Ne}$. This result is attributable to the conversion of ${ }^{14} \mathrm{~N}$ into ${ }^{22} \mathrm{Ne}$ via $\alpha$ captures during thermal pulses. The efficient destruction of ${ }^{19} \mathrm{~F}$ occurs by the HBB in the 6- and 8-M $\mathrm{M}_{\odot}$ models. The same results was shown in Lugaro et al. (2008), in which their $M \gtrsim 3 \mathrm{M}_{\odot}$ models with $[\mathrm{Fe} / \mathrm{H}]=-2.3$ destroy ${ }^{19} \mathrm{~F}$ by $\mathrm{HBB}$. The resulting abundances are $[\mathrm{F} / \mathrm{Fe}] \lesssim 0$ in the yields of $6-$ and $8-\mathrm{M}_{\odot}$ models. On the other hand, enhancements of ${ }^{19} \mathrm{~F}$, which is produced in the He convective shell during thermal pulses, are found. The abundances in the calculated yields are $[\mathrm{F} / \mathrm{Fe}]=3.07,2.20$ and 1.92 for the models of 2-, 3- and 4$\mathrm{M}_{\odot}$, respectively, assuming $[\mathrm{Fe} / \mathrm{H}]=-3$. Straniero et al. (2004) showed that $[\mathrm{F} / \mathrm{Fe}]=3.15$ in the $1.5-\mathrm{M}_{\odot}$ model with $[\mathrm{Fe} / \mathrm{H}] \simeq-2.6$. The present result of the $2-\mathrm{M}_{\odot}$ model is comparable to that in Straniero et al. (2004).

The calculated yields are compared with the results of Campbell \& Lattanzio (2008), in which the evolution and nucleosynthesis in the models with $0.85-, 1-, 2$ - and 3- $\mathrm{M}_{\odot}$ and $[\mathrm{Fe} / \mathrm{H}]=-3$ were followed. Campbell \& Lattanzio (2008) did not find the HIE in their $2-\mathrm{M}_{\odot}$ model. This may be due to their strict application of Schwarzschild criterion to the convective boundary. In contrast to theirs, we assume that the radiative mesh next to convective boundary is further included as the mixed layer, although we also adopt the Schwarzschild criterion for determining convective boundaries. Therfore, we find the HIE in the $2-\mathrm{M}_{\odot}$ model. The yields by Campbell \& Lattanzio (2008) showed a large difference with those in the present results. The main reason for such large differences comes from the adopted mass loss formula. They used the one developed by Vassiliadis \& Wood (1993). The formula gives mass loss rate lower than the presently used Blöcker's rate (Blöcker 1995). This fact is easily found in their remnant masses for each model. For example, in the case of $2-\mathrm{M}_{\odot}$ model their remnant mass was reported as 1.038$\mathrm{M}_{\odot}$, although we obtain $0.66-\mathrm{M}_{\odot}$ as seen in Table 1 . Their low mass models which leave higher remnant mass are likely to experience HBB. Campbell \& Lattanzio (2008) showed the large enhancements of ${ }^{14} \mathrm{~N}$ and ${ }^{23} \mathrm{Na}$ in their 2and $3-\mathrm{M}_{\odot}$ models, since the models experience the larger number of thermal pulse cycle due to the longer period of AGB phases. However, we only obtain small yields of them due to inefficient activation of $\mathrm{HBB}$ in our $3-\mathrm{M}_{\odot}$ model.

In order to compare the abundance pattern in the yield ejected from the AGB models with that observed in extremely metal-poor stars, we take into account a dilution in the surface convection zone of the low-mass binary companion which is now observed. The dilution factor is defined as $f_{\text {dil }}=\log _{10} M_{\text {mix }} / M_{\text {acc }}$, where $M_{\text {acc }}$ is the mass accreted from an AGB star to the secondary and $M_{\text {mix }}$ is the secondary envelope mass including the accreted gas with AGB compositions (Thompson et al. 2008). We attempt to explain the abundance pattern of CS 29528041 which has the metallicity of $[\mathrm{Fe} / \mathrm{H}]=-3.3$ (Sivarani et al. 2006). The observed ratio of $[\mathrm{C} / \mathrm{N}]=-1.48$ indicates that an intermediate-mass star can be a donor. The abundance pattern in the yield of the $6-\mathrm{M}_{\odot}$ AGB model is compared with that of CS 29528-041 in the top panel of Figure 5 . In this case we use $f_{\text {dil }}=0.66$ dex to reproduce the observed patterns, although we find slight underproduction of $\mathrm{N}$ and overproduction of $\mathrm{Al}$. The Li abundance in the transferred material is close to the Spite plateau value. As the result, the diluted abundance of $\mathrm{Li}$ is still high relative to the observation, but the extra mixing processes may act the accreted material and decrease to the observed value during the long lifetime of the low-mass secondary (Stancliffe et al. 2007; Thompson et al. 2008). Unfortunately, the carbon isotopic ratio was not observed in CS 29528-041, but the present investigation predicts ${ }^{12} \mathrm{C} /{ }^{13} \mathrm{C}=11.8$.

In addition, the abundance patterns of hot turn-off star CS 29497-030 are compared with the yields calculated from the models in the bottom panel of Figure 5. We note that, for this star, the metallicities analyzed by different authors show a diversity $([\mathrm{Fe} / \mathrm{H}]=-2.16$ to -2.8$)$. In this investigation we assume that CS 29497-030 has the lower metallicity of -2.8 (Sivarani et al. 2004) or -2.57 

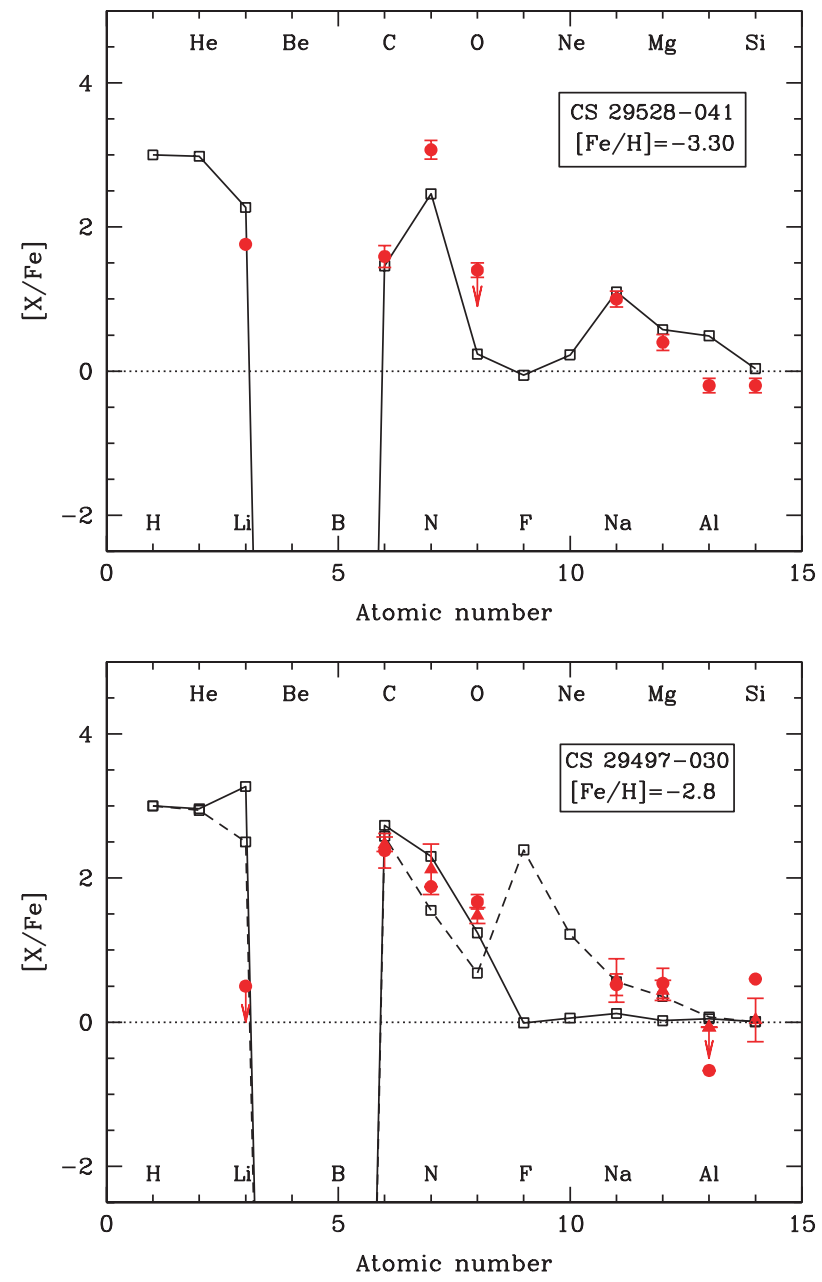

Figure 5 Comparison of the abundance patterns observed in extremely metal-poor stars with the yields ejected from the models $([\mathrm{Fe} / \mathrm{H}] \simeq-3)$. In the top panel the abundance pattern (filled circles) in CS 29528-041 observed by Sivarani et al. (2006) is compared with the yield from the 6-M $\mathrm{M}_{\odot}$ model. The only upper limit of oxygen abundance is given from the observation. In the bottom panel the abundance patterns of CS 29497-030 are represented by filled circles (Sivarani et al. 2004) and filled triangles (Ivans et al. 2005) and are compared with the yields ejected from the models of 1- and $2-\mathrm{M}_{\odot}$ (solid and dashed lines, respectively).

(Ivans et al. 2005). This star shows binarity and an enrichment of the $s$-process elements. Therefore, it is likely that the primary companion evolves AGB phase. The detailed comparison of the observed abundance patterns with yields from the models with masses of 1- and 2- $\mathrm{M}_{\odot}$ shows a relatively good agreement with $\mathrm{CNO}, \mathrm{Na}, \mathrm{Mg}$ and $\mathrm{Si}$. Therefore, it is expected that the abundance pattern of CS 29497-030 can be reproduced by the TP-AGB model with about $1.5-\mathrm{M}_{\odot}$. The enrichment of heavy neutroncapture elements will be explained by the $s$-process in the ${ }^{13} \mathrm{C}$ pocket during the interpulse phases (Ivans et al. 2005). For this fitting we also adopt $f_{\text {dil }}=0.66$ dex. The observed abundance of $\mathrm{Li}$ is very deficient with respect to the predicted ones and Spite plateau value. This fact may be the signature of efficient ${ }^{7} \mathrm{Li}$ destruction by e.g. thermohaline mixing (Stancliffe et al. 2007).

\section{Concluding Remarks}

We calculate the detailed evolution and nucleosynthesis of extremely metal-poor $([\mathrm{Fe} / \mathrm{H}] \simeq-3)$ star models with 1-8 $\mathrm{M}_{\odot}$. The models of $M<3 \mathrm{M}_{\odot}$ are affected by the occurrence of HIE and predict the production of large amounts of ${ }^{7} \mathrm{Li}$ and ${ }^{14} \mathrm{~N}$. We confirm that the higher mass AGB models also create ${ }^{7} \mathrm{Li}$ by the HBB. Using the present formulation of mass loss rate, the ${ }^{7} \mathrm{Li}$ abundances in the yields of the AGB models with 1,2, 4 and $8 \mathrm{M}_{\odot}$ exceed the primordial value. This result indicates that the Li-enriched low-metallicity dwarfs might be found out if there exists a binary system consisting of an AGB star with, especially, $1 \lesssim M / M_{\odot}<3$. This prediction, however, becomes only true if the extra mixing processes (e.g. atomic diffusion, rotation-induced mixing and thermohaline mixing) in the low-mass secondary do not work efficiently during its long life. The ${ }^{7} \mathrm{Li}$ yields from higher mass AGB stars are sensitive to the mass loss history of AGB evolution. If the mass loss rate is not so high at the early phase of TPAGB, the ejected ${ }^{7} \mathrm{Li}$ amounts are expected to decrease. This may have a relationship with the small amount of $\mathrm{Li}$ depletion observed in extremely metal-poor stars (Aoki et al. 2008). The abundance patterns of yields ejected from the calculated AGB models are compared with those of two (extremely) metal-poor stars, CS 29528-041 and CS 29497-030. We demonstrate that the present results can explain the observational patterns.

Finally, we note that the proper inclusion of convectivereactive feedback at the occurrence of the HIE may be important to follow the flash phenomena with huge energy release as shown in this investigation. In order to resolve this issue, multi-dimensional simulation is considered to be an appropriate way forward (Woodward et al. 2008). In contrast, in a one-dimensional approach the coupling of stellar structure equations to changes in chemical composition (by mixing and nuclear burning) is necessary to treat the energy release, which significantly affects the structure change (Stancliffe 2006). The investigation of this direction will also be needed in future work.

\section{Acknowledgments}

The author is grateful to the anonymous referee for the helpful comments. The author would like to thank Prof. Roberto Gallino most warmly for his kind hospitality during the stay in Torino and for fruitful discussions on this work. The author appreciates the use of SAGA database (Suda et al. 2008). This work has been supported by the Grant-in-Aid for Young Scientists (B) (17740163).

\section{References}

Abia, C. \& Isern, J., 1997, MNRAS, 289, L11

Alexander, D. R. \& Ferguson, J. W., 1994, ApJ, 437, 879

Anders, E. \& Grevesse, N., 1989, GeCoA, 53, 197

Angulo, C. et al., 1999, NuPhA, 656, 3

Aoki, W., Barklem, P., Beers, T. C., Christlieb, N. \& Inoue, S., 2008, AIPC, 1016, 37

Bao, Z. Y., Beer, H., Käppeler, F., Voss, F., Wisshak, K. \& Rauscher, T., 2000, ADNDT, 76, 70

Blöcker, T., 1995, A\&A, 297, 727 
Cameron, A. G. W. \& Fowler, W. A., 1971, ApJ, 164, 111

Campbell, S. W. \& Lattanzio, J. C., 2008, A\&A, 490, 769

Caughlan, G. R. \& Fowler, W. A., 1988, ADNDT, 40, 283

Christlieb, N. et al., 2002, Natur, 419, 904

Cristallo, S., Straniero, O., Lederer, M. T. \& Aringer, B., 2007, ApJ, 667,489

Forestini, M. \& Charbonnel, C., 1997, A\&AS, 123, 241

Frebel, A. et al., 2005, Natur, 434, 871

Frebel, A., Collet, R., Eriksson, K., Christlieb, N. \& Aoki, W., 2008, ApJ, 684, 588

Fujimoto, M. Y., Ikeda, Y. \& Iben, I. J., 2000, ApJ, 529, L25

Garcia-Berro, E., Ritossa, C. \& Iben, I. J., 1997, ApJ, 485, 765

Herwig, F., 2004, ApJS, 155, 651

Herwig, F., 2005, ARA\&A, 43, 435

Iben, I. J., Ritossa, C. \& Garcia-Berro, E., 1997, ApJ, 489, 772

Iglesias, C. A. \& Rogers, F. J., 1996, ApJ, 464, 943

Ivans, I. I., Sneden, C., Gallino, R., Cowan, J. J. \& Preston, G. W., 2005, ApJ, 627, L145

Iwamoto, N., Kajino, T., Mathews, G. J., Fujimoto, M. Y. \& Aoki, W., 2004, ApJ, 602, 377

Lambert, D. L., Smith, V. V., Busso, M., Gallino, R. \& Straniero, O., 1995, ApJ, 450, 302

Lugaro, M. et al., 2008, A\&A, 484, L27

Marigo, P., 2002, A\&A, 387, 507

Mowlavi, N., 1999, A\&A, 350, 73

Plez, B., Smith, V. V. \& Lambert, D. L., 1993, ApJ, 418, 812

Rauscher, T. \& Thielemann, F.-K., 2000, ADNDT, 75, 1

Richard, O., Michaud, G. \& Richer, J., 2005, ApJ, 619, 538
Ritossa, C., Garcia-Berro, E. \& Iben, I. J., 1996, ApJ, 460, 489

Sackmann, I.-J. \& Boothroyd, A. I., 1992, ApJ, 392, L71

Sivarani, T. et al., 2004, A\&A, 413, 1073

Sivarani, T. et al., 2006, A\&A, 459, 125

Smith, V. V. \& Lambert, D. L., 1989, ApJ, 345, L75

Smith, V. V. \& Lambert, D. L., 1990, ApJ, 361, L69

Spergel, D. N. et al., 2003, ApJS, 148, 175

Spergel, D. N. et al., 2007, ApJS, 170, 377

Spite, F. \& Spite, M., 1982, A\&A, 115, 357

Stancliffe, R. J., 2006, MNRAS, 370, 1817

Stancliffe, R. J. \& Glebbeek, E., 2008, MNRAS, 389, 1828

Stancliffe, R. J., Glebbeek, E., Izzard, R. G. \& Pols, O. R., 2007, A\&A, 464, L57

Straniero, O., Cristallo, S., Gallino, R. \& Dominguez, I., 2004, MmSAI, 75, 665

Suda, T., Aikawa, M., Machida, M. N., Fujimoto, M. Y. \& Iben, I. J., 2004, ApJ, 611, 476

Suda, T. et al., 2008, PASJ, 60, 1159

Talon, S. \& Charbonnel, C., 1998, A\&A, 335, 959

Thompson, I. B. et al., 2008, ApJ, 677, 556

Tumlinson, J., 2007, ApJ, 665, 1361

Uttenthaler, S., Lebzelter, T., Palmerini, S., Busso, M., Aringer, B. \& Lederer, M. T., 2007, A\&A, 471, L41

Vassiliadis, E. \& Wood, P. R., 1993, ApJ, 413, 641

Wood, P. R., Bessell, M. S. \& Fox, M. W., 1983, ApJ, 272, 99

Woodward, P., Herwig, F., Porter, D., Fuchs, T., Nowatzki, A. \& Pignatari, M., 2008, AIPC, 990, 300 